18 ONE PAGE USER FRIENDLY PROFORMA DELIVERS DRAMATIC IMPROVEMENTS IN HEART FAILURE MANAGEMENT IN A BUSY DISTRICT GENERAL HOSPITAL

${ }^{1}$ Clare Coyle*, ${ }^{2}$ Baltazar Nyathi, ${ }^{3}$ Simon Woldman, Mihir Sanghvi, Lindsey Iles, Sandy Gupta, Debashish Das. ' Barts Health; ${ }^{2}$ Queen Mary University; ${ }^{3}$ Barts Heart Centre; *Presenting Author

\subsection{6/heartjnl-2016-309890.18}

Background Heart failure affects almost one million people in the UK with survival rates comparable to or worse than many cancers. Recent publications of the NICOR National Heart Failure Audit have focused attention on the need for improvement. This challenge is particularly acute in busy district general hospitals in London such as Whipps Cross University Hospital.

Objective We aimed to investigate if the implementation of a simple one-page user friendly Whipps Cross Heart Failure Improvement Proforma- the 'WHIP form' in all medical wards could help improve the management of patients admitted with heart failure against standard quality measures.

Methods The 'WHIP form' was introduced and implemented in all medical wards supported with a one-day educational seminar and a new dedicated heart failure email service.

Results Between June to September 2015, 106 patients with a primary admission diagnosis of heart failure were enrolled and managed using the 'WHIP form'. Inpatient mortality remained stable at $11.3 \%$ with an average hospital stay of 13.5 days. The 30-day readmission rate halved from $14 \%$ to $7 \%$. Patients with documented left ventricular systolic dysfunction on Echocardiogram had significant improvements in the prescription of prognostic medication on discharge: ACEi/ARBs prescription increased from $78 \%$ from $88 \%$ [10\% improvement]. BBlockers prescription increased from $68 \%$ to $95 \%$ [27\% improvement].

Conclusion The initiation of an "easy to use" one page heart failure management proforma led to a dramatic reduction in 30 -day readmission rates and significant increase in the prescription of prognostically important ACE inhibitors and Bblockers. If the reductions in 30-day readmissions are sustained, we estimate that our cost neutral intervention could translate to yearly savings of nearly $£ 80 \mathrm{~K}$ for Whipps Cross University Hospital alone.

\section{TIME FROM SYMPTOMS ONSET TO DIAGNOSIS AND OPTIMUM THERAPY; DOES IT RELATE TO OUTCOME IN HEART FAILURE PATIENTS?}

Ali Wahab*, Haqeel Jamil, John Gierula, Maria Paton, Roo Byrom-Goulthorp, Emma Sunley, Sarah Winsor, Richard Cubbon, Mark Kearney, Klaus Witte. NHS; *Presenting Author

\subsection{6/heartjnl-2016-309890.19}

Introduction Current National Institute of Health and Care Excellence (NICE) guideline recommendations on diagnosing heart failure (HF) in primary care include the measurement of serum natriuretic peptide concentrations (NP). NP concentrations are raised in HF related to severity. In addition to advising a referral above an 'intermediate' level (to be seen within 6 weeks), the guidelines suggest that patients with 'high' levels should be seen more urgently (within 2 weeks). This prospective service evaluation aimed to provide evidence to support this challenging requirement by exploring the impact of time

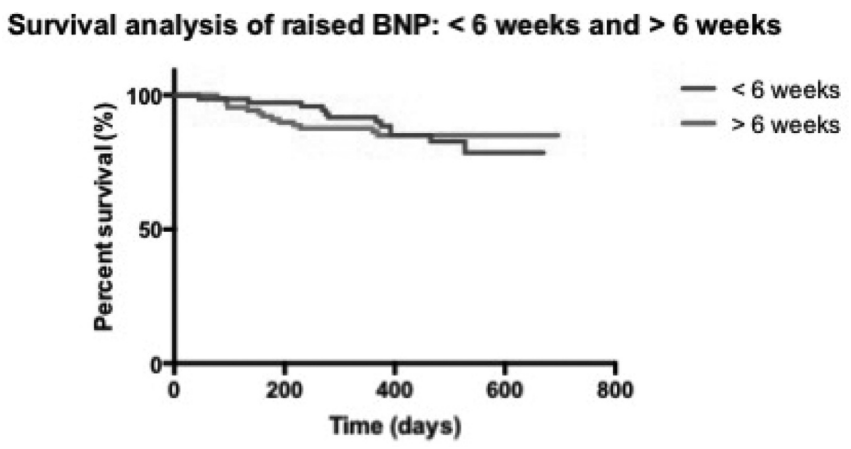

Abstract 19 Figure 1 Kaplan Meier curve showing no significant difference in mortality for patients with raised BNP seen within and after 6 weeks $(p=0.49)$

from symptoms onset to diagnosis and optimum treatment on subsequent long-term mortality in patients with $\mathrm{HF}$ due to left ventricular systolic dysfunction (LVSD).

Methods We recorded date of symptom onset, referral, diagnosis and subsequent mortality in 206 patients newly diagnosed with HF due to LVSD seen in clinic following a NP test over twelve months from May 2012. The primary investigator (AW) was blinded to the NP result. Patients were initially divided into groups 'high' and 'intermediate' and then by whether they were seen within NICE-stipulated timeframes $(\mathrm{n}=71)$ or not $(\mathrm{n}=109)$. Due to a limited number of events, outcomes have been pooled using the 6 weeks cut-off. All-cause mortality was plotted on Kaplan-Meier curves and logistic regression was used to determine whether times between points on the pathway and being seen within 6 weeks featured in models including other known predictors of mortality.

Results After a mean follow-up time of 411 days (range (219255 days)) 24 patients with a raised NP result had died (15\%). Mean clinic visit time from the date of the blood test was 60 days (range (57-63 days)). Kaplan-Meier curves indicated no difference in mortality for patients seen within 6 weeks according to NP levels and those seen later than 6 weeks $(\mathrm{p}=0.49)$.

Conclusion In patients presenting to their primary care physician with symptoms possibly due to heart failure, subsequently confirmed to have LVSD, there is no difference in outcomes if they are seen within 6 weeks or not. The guideline that patients with symptoms possibly due to $\mathrm{HF}$ and a raised NP level should be seen within 6 weeks, has the potential to lead to great pressure on all outpatient cardiology services yet seems to have no impact on mortality. Whether hospitalisation is avoided by urgent referral remains to be assessed.

\section{FACTORS WHICH IMPACT ON MORTALITY AND READMISSION IN PATIENTS WITH HEART FAILURE: REAL WORLD LONGITUDINAL DATA}

${ }^{1}$ Yang Chen*, ${ }^{2}$ Markus Sikkel, ${ }^{3}$ Jaymin Shah. ${ }^{1}$ Barts Health NHS Trust; ${ }^{2}$ Imperial College Healthcare NHS Trust; ${ }^{3}$ London North West Healthcare NHS Trust; *Presenting Author

\subsection{6/heartjnl-2016-309890.20}

Background Heart failure remains challenging to manage and treat globally. Much of the data on its prognosis stems from clinical trials, where cohorts are often younger and less sick than the groups of patients usually treated in hospitals and 